\title{
The Value of Different Radiological Modalities in Assessment of Spontaneous Pneumomediastinum: Case Review and Diagnostic Perspective
}

\author{
Poonam Singh Jariwala, MBBS ${ }^{1}$ Kaarthigeyan Kalaniti, MD ${ }^{1,2}$ Neil Wonko, MD ${ }^{1,2}$ \\ Sibasis Daspal, MD ${ }^{1,2}$ Veronica Mugarab Samedi, MD $^{1,2}$ \\ ${ }^{1}$ Department of Pediatrics, University of Saskatchewan, Saskatoon, Canada \\ 2 Department of Pediatrics, Royal University Hospital, Saskatoon, SK, Canada \\ Address for correspondence Veronica M. Samedi, MD, Department of \\ Pediatrics, University of Saskatchewan, Saskatoon, SK, Canada \\ (e-mail: vmsamedi@gmail.com; mougarab@hotmail.com).
}

Am J Perinatol Rep 2019;9:e72-e75.

Abstract
Keywords
- neonate
- pneumomediastinum
- lung ultrasound
- chest radiography
- computerized
tomography scan

Pneumomediastinum (PM), or a mediastinal air leak, is a known morbidity in neonates. Among predisposing factors, the most significant are mixed lung diseases, such as pneumonia or meconium aspiration syndrome, with coexisting atelectasis and airway obstruction. We report an unusual presentation of significant spontaneous pneumomediastinum in a term newborn delivered by elective cesarean section with no history of active resuscitation at birth.

Timely diagnosis of SPM is important for both management and prognosis. We believe that lung ultrasound (LUS) is a promising screening tool for early detection of PM in neonates, and could help avoid unnecessary exposure to radiation in delicate neonates. More simultaneous studies comparing chest X-ray (CXR), LUS, and computed tomography $(C T)$ scans may help to prove the utility value of point-of-care ultrasounds as a primary diagnostic tool in identifying this morbidity.

Pneumomediastinum (PM), or a mediastinal air leak, is a known morbidity in neonates. Among predisposing factors, the most significant are mixed lung diseases, such as pneumonia or meconium aspiration syndrome, with coexisting atelectasis and airway obstruction. ${ }^{1}$ We report an unusual presentation of significant spontaneous pneumomediastinum (SPM) in a term newborn delivered by elective cesarean section with no history of active resuscitation at birth.

\section{Case description}

A $3.45 \mathrm{~kg}$ male child was born to a G4, P3 healthy mother by repeat elective $\mathrm{C}$-section at 38 weeks of gestation. The antenatal period was uneventful. Mother had protective serologies and no known exposures during pregnancy. Apgar's scores were 6,8 , and 10 at 1,5 , and 10 minutes, respectively, and the infant did not require any resuscitation maneuvers, namely, positive pressure ventilation. Within the 1 st hour of life, the infant developed grunting with tachypnea and desaturations
$\left(\mathrm{SpO}_{2}\right.$ to $50 \%$ in $\left.\mathrm{FiO}_{2} 21 \%\right)$. His blood gases showed metabolic acidosis and chest X-ray (CXR) showed air-bronchograms in both lungs with an ill-defined cardiac border (-Fig. 1). His respiratory status rapidly worsened needing intubation, surfactant, and transport to our tertiary care center.

CXR was repeated upon arrival at the tertiary unit showed hyperlucency in superior medial right hemithorax which was new from prior image, situated in previous areas of paratracheal opacities. Subsequent CXRs showed that the previously described hyperlucent shadow became more evident bilaterally (-Fig. 2). Differential diagnosis at this stage included possible congenital pulmonary airway malformation (CPAM) versus pneumomediastinum. Bedside lung ultrasound (LUS) on day-2 of life showed the classic "bar-code" sign suggestive of an air-leak rather than air-filled cystic mass (-Figs. 3, 4). Computerized tomography (CT) scan of the chest was requested to confirm the presence of a large extensive pneumomediastinum, expanding in the mediastinum and causing soft tissue gas to extend into the right side of the neck ( - Fig. 5). The rest of the received January 29, 2019 accepted

February 5, 2018
DOI https://doi.org/ $10.1055 / \mathrm{s}-0039-1683862$. ISSN 2157-6998.
Copyright (C) 2019 by Thieme Medical Publishers, Inc., 333 Seventh Avenue, New York, NY 10001, USA. Tel: +1(212) 584-4662.
License terms

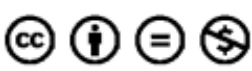




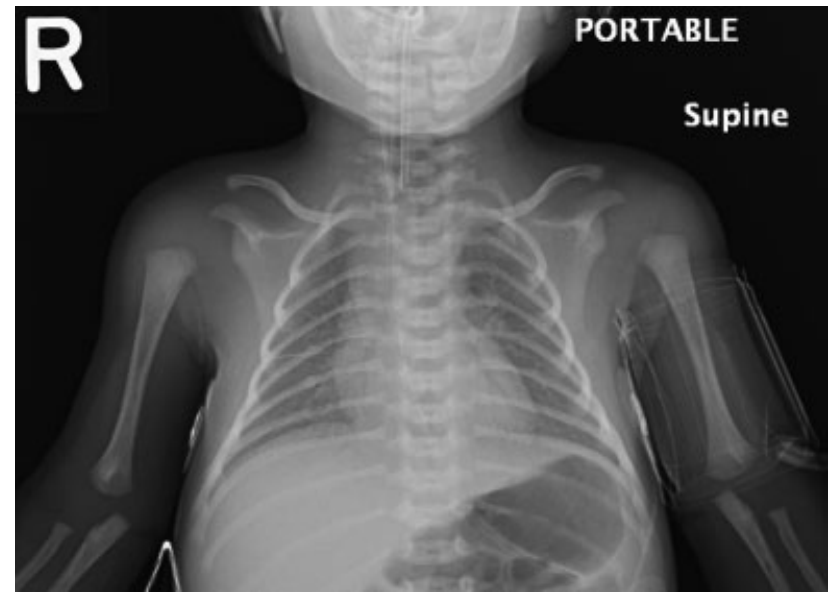

Fig. 1 CXR-early alveolar changes showing bilateral air-bronchograms on day 1 of life. CXR, chest X-ray.

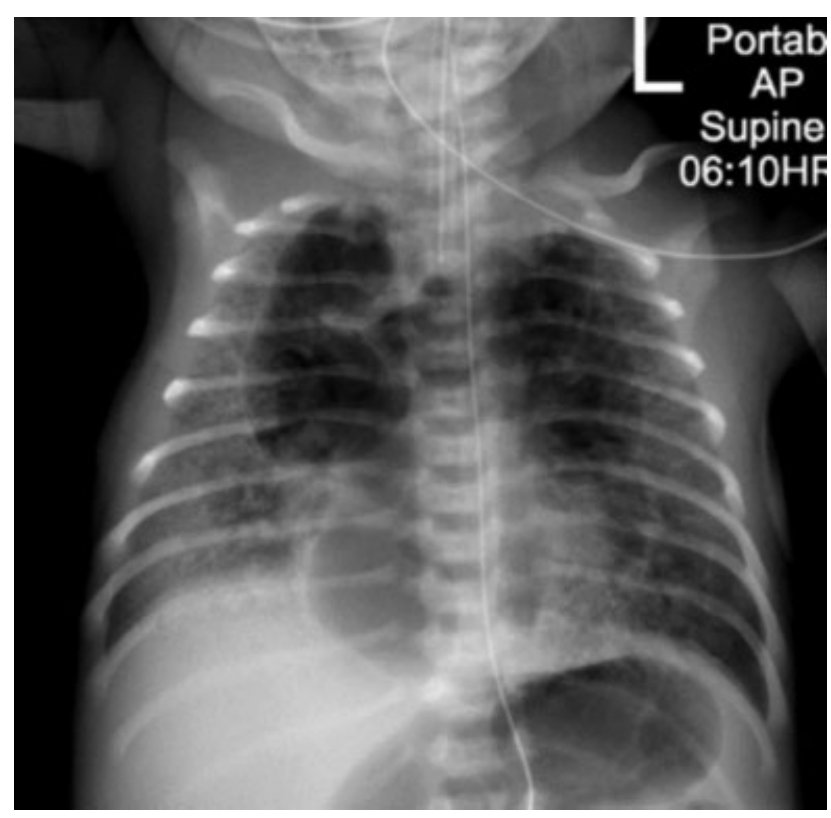

Fig. 2 CXR-bilateral hyperlucent shadows in superior medial hemithoraces over the area of pneumomediastinum on day 2 of life. AP, anteroposterior; CXR, chest X-ray.

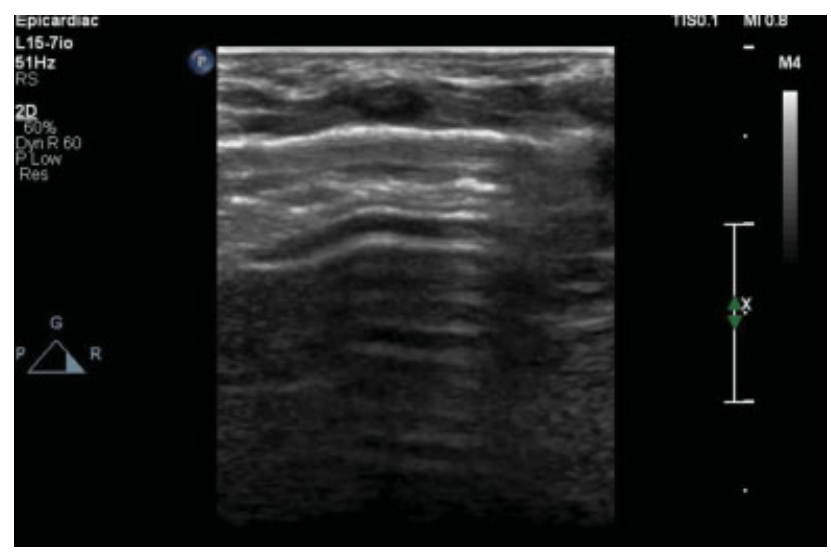

Fig. 3 LUS-presence of multiple "A" lines, absence of "B" lines and no pleural shimmering. LUS, lung ultrasound.

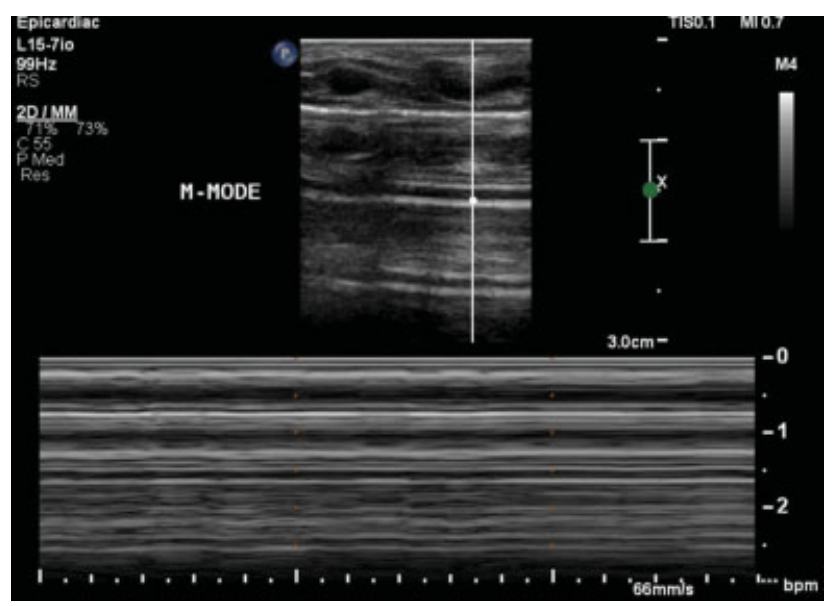

Fig. 4 LUS-M-mode with classic "bar-code" sign over the area of pneumomediastinum. LUS, lung ultrasound; M-mode, motion over time.

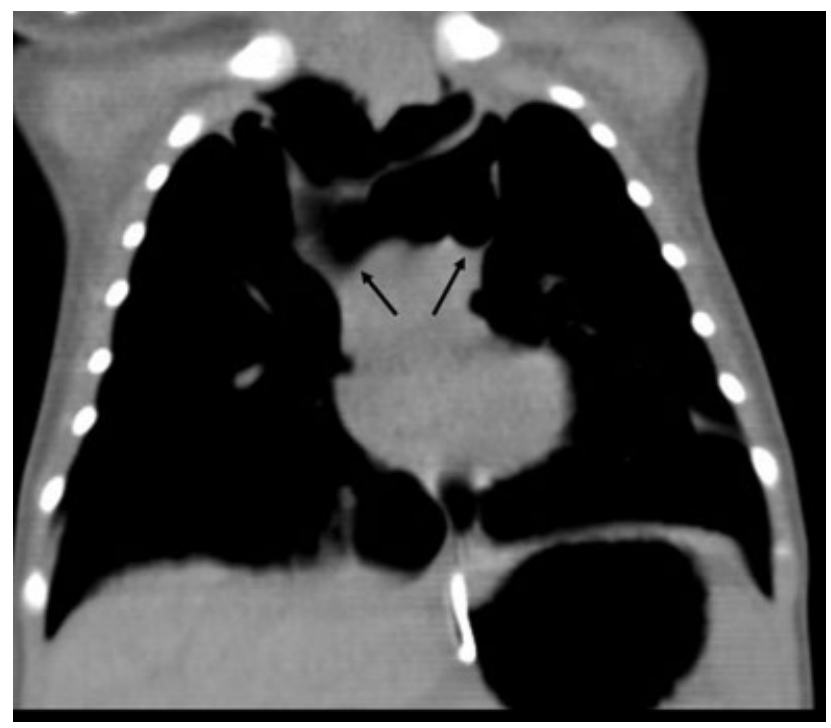

Fig. 5 CTscan-showing expanding air in the mediastinum (arrows) causing adjacent atelectasis within the medial aspects of both right and left upper lobes of lungs by day 3 of life. CT, computed tomography.

clinical course was straightforward; the infant required respiratory support for a total of 6 days. CXR done on day- 8 of life showed complete resorption of the pneumomediastinum. No other complications were noted during the neonatal intensive care unit (NICU) stay.

\section{Discussion}

SPM)in a term baby without assisted ventilation or known underlying disease is uncommon. Neonatal PM occurs in approximately 2.5 per 1,000 live births. ${ }^{2}$ Various causes of PM are described in the literature, namely, airway obstruction as seen in foreign body aspiration, iatrogenic causes like mechanical ventilation, infections, obstructive lung disease, such as asthma, following chest trauma, Valsalva's maneuvers during vomiting or convulsion or as a result of toxic effects of smoking, and the weakness of tissue (e.g., anorexia nervosa). In SPM, the underlying lung is healthy, and the air leak is thought to be atraumatic. ${ }^{3}$ If a pressure gradient develops between the 
alveoli and surrounding tissues, an alveolar rupture can result. The pressure gradient may be caused by either over inflation of alveolus or decrease in the surrounding interstitial pressures. The air leak can diffuse into interstitial tissue, mediastinum, and subcutaneous tissue. ${ }^{3,4}$

Scarce literature is found about neonates with PM. In a series of nine cases of neonates with PM published, from University Children's Hospital, Zurich, Switzerland, more than half of the neonates had no obvious reasons, ${ }^{5}$ similar to our patient who had no history of eventful labor or positive-pressure ventilation at birth. The mechanical events are known causes of air leaks in older infants, whereas the exact etiology in neonates remains unclear. The initial presentation of SPM in newborns is respiratory distress irrespective of the underlying cause. In the above case series, two neonates were premature and seven were term, and all presented with signs of respiratory distress. There was no significant difference to the rate of occurrence of PM based on mode of delivery, that is, vaginal and Csection deliveries. All neonates were vigorous at birth and none of them required resuscitation with bag-mask ventilation as in our case. Our child, however, received a dose of surfactant later which was not documented in the case series reported. However, during hospitalization in the intensive care unit, two neonates from the case series deteriorated and required mechanical ventilation for 3 and 4 days, respectively. The case series also report two other neonates that needed CPAP (continuous positive airway pressure) initially before arriving to the intensive care unit, where the diagnosis of PM was confirmed by CXR. Only one of the preterm neonates, in the case series mentioned, underwent intubation and required ventilator support. The causes of PM identified in the case series ranged from Valsalva's manuvere caused by convulsions, pulmonary infection due to maternal infection and possible barotrauma in premature neonate. The duration of hospitalization stay ranged from 3 to 13 days where as in our case it was 9 days in total.

In infants, a pathologic finding seen in the CXR diagnostic of PM is the classic "spinnaker sail sign" also known as "angel wing" sign. The lobe/lobes of the thymus are displaced superiorly and laterally, separated from the heart and mediastinal structures by air in the mediastinum. ${ }^{6}$ Typical radiological signs of PM include the "continuous diaphragm sign" (interposition of air between the pericardium and the diaphragm which becomes visible in the central mediastinal part) and linear bands of mediastinal air paralleling the left side of the heart and the descending aorta (pleura is shown as a fine opaque line) with extension superiorly along the great vessels into the neck. Lateral CXR is important and often more sensitive than the anteroposterior view in making the diagnosis. However, CXR alone can underestimate small pneumomediastinum even if the lateral view is obtained. The benefits of bedside LUS include low-cost, no threat of radiation damage, and adequate reliability. Accuracy of LUS in detecting air-leaks, mostly pneumothoraxes, has been well described in the literature. $^{7,8}$ The four most important sonographic signs used to diagnose pneumothorax are the presence of lung point, absence of lung sliding, B-lines and lung pulse, and the sensitivity and specificity of lung point in diagnosing pneumothorax are 66 and $100 \%$, respectively. ${ }^{9,10}$ However, there are very limited reports related with the use of LUS in detecting PM, especially in infants.

Chest CT scan is traditionally considered superior to chest radiography and can be used to confirm the diagnosis even with a minimal amount of air. ${ }^{11}$ CXRs can be negative in up to $30 \%$ of cases of SPM. ${ }^{11}$ There is also a significant time lag between the emergence of clinical and radiological signs. ${ }^{12}$ In our case, a possibility of congenital lobar emphysema (CLE) was entertained as initial roentogram showed unilateral hypertranslucency and therefore, a CT scan was performed to exclude other possibilities. At the same time, availability, cost, possible need for sedation and exposure to radiation are known disadvantages of CT scan in neonates.

PM in children and neonates has a good prognosis ${ }^{5,10}$ and early diagnosis of this morbidity is important. While initial presentation and radiographic findings for PM, CLE, and CPAM look similar, management and prognosis for these conditions totally diverge. Some newborns with PM may require ventilator support, but respiratory distress usually subsides within 7 to 10 days, while CPAM patients often require prolonged observation and even possible surgical intervention.

\section{Conclusion}

Timely diagnosis of SPM is important for both management and prognosis. We believe that LUS is a promising screening tool for early detection of PM in neonates, and could help avoid unnecessary exposure to radiation. More simultaneous studies comparing CXR, LUS, and CT scans may help to prove the utility value of point-of-care ultrasounds as a primary diagnostic tool in identifying this morbidity.

\section{Conflict of Interest}

None.

\section{References}

1 Carey B. Neonatal air leaks: pneumothorax, pneumomediastinum, pulmonary interstitial emphysema, pneumopericardium. Neonatal Netw 1999;18(08):81-84

2 Steele RW, Metz JR, Bass JW, DuBois JJ. Pneumothorax and pneumomediastinum in the newborn. Radiology 1971;98(03): 629-632

3 Macklin CC. Transport of air along sheaths of pulmonic blood vessels from alveoli to mediastinum: clinical implications. Arch Intern Med (Chic) 1939;64:913-926

4 Macklin MT, Macklin CC. Malignant interstitial emphysema of the lungs and mediastinum as an important occult complication in many respiratory diseases and other conditions: an interpretation of the clinical literature in the light of laboratory experiment. Medicine (Baltimore) 1944;23(04):281-358

5 Hauri-Hohl A, Baenziger O, Frey B. Pneumomediastinum in the neonatal and paediatric intensive care unit. Eur J Pediatr 2008; 167(04):415-418

6 Chalumeau M, Le Clainche L, Sayeg N, et al. Spontaneous pneumomediastinum in children. Pediatr Pulmonol 2001;31(01):67-75 
7 Ng L, Saul T, Lewiss RE. Sonographic evidence of spontaneous pneumomediastinum. Am J Emerg Med 2013;31(02):462. e3-462.e4

8 Russo A, Del Vecchio C, Zaottini A, Giangregorio C. Role of emergency thoracic ultrasonography in spontaneous pneumomediastinum. Two case report. G Chir 2012;33 (8-9): 285-296

9 Jung AY, Yang I, Go HS, et al. Imaging neonatal spontaneous pneumomediastinum using ultrasound. J Med Ultrason 2001 2014;41 (01):45-49
10 Okada M, Adachi H, Shibuya Y, Ishikawa S, Hamabe Y. Diagnosis and treatment of patients with spontaneous pneumomediastinum. Respir Investig 2014;52(01):36-40

11 Kaneki T, Kubo K, Kawashima A, Koizumi T, Sekiguchi M, Sone S. Spontaneous pneumomediastinum in 33 patients: yield of chest computed tomography for the diagnosis of the mild type. Respiration 2000;67(04):408-411

12 Panacek EA, Singer AJ, Sherman BW, Prescott A, Rutherford WF. Spontaneous pneumomediastinum: clinical and natural history. Ann Emerg Med 1992;21(10):1222-1227 